\title{
Classificação numérica e modelo digital de elevação na caracterização espacial de atributos dos solos
}

\begin{abstract}
João F. da Silva Júnior ${ }^{1}$, Diego S. Siqueira ${ }^{1}$, José Marques Júnior ${ }^{1} \&$ G ener T. Pereira ${ }^{1}$
RESU M O

U ma das necessidades da agricultura moderna é a predição da variabilidade espacial dos atributos dos solos em escalas mais detalhadas, visando o gerenciamento sustentável e a otimização das práticas de manejo dos solos. Associada ao conhecimento da variabilidade dos atributos do solo e ao mapeamento das formas do relevo, a modelagem matemática tem auxiliado no planejamento agrícola. Objetivou-se então, no presente trabalho, caracterizar a variabilidade espacial de atributos físicos e químicos de Latossolo e Argissolo utilizando-se a classificação numérica e o modelo digital de elevação em duas áreas, identificadas por duas pedoformas distintas: pela convexa, para o Latossolo (158 ha) e por meio da linear, para o Argissolo (172 ha). Foram retiradas 53 amostras no Latossolo e 57 amostras no Argissolo e realizada análise multivariada de agrupamentos dos atributos estudados a partir de suas distâncias euclidianas. Esta análise caracterizou, por meio de dendogramas e juntamente com os modelos digitais de elevação para os diferentes solos, grupos mais homogêneos em Argissolo e menos homogêneos para o Latossolo em pedoforma convexa. Esses métodos quantitativos mostraram que as pedoformas condicionaram o padrão espacial dos atributos dos solos.
\end{abstract}

Palavras-chave: análise multivariada, agricultura precisão, mapeamento híbrido, relação solo-paisagem

\section{Numerical classification and digital elevation model to spatial characterization of soil attributes}

\begin{abstract}
A B ST RACT
O ne of needs of modern agriculture is the prediction of spatial variability of soil properties at more detailed scales for sustainable management and optimization of management practices. The mathematical model associated with knowledge of variability of soil attributes and mapping of relief forms has helped in agricultural planning. In this regard the aim of this study was to characterize the spatial variability of physical and chemical properties of 0 xisols and Ultisols using numerical classification and the digital elevation model. Two distinct landforms: convex for the 0 xisol (158 ha) and linear for the Ultisol (172 ha). 53 samples from the 0 xisol and 57 samples from the UItisol were taken. Multivariate analysis of clusters of attributes studied from their euclidean distances was performed. This analysis by dendograms along with digital elevation models for different soils characterized was more homogeneous in Ultisol groups, and less homogeneous for the Oxisol in convex landform. These quantitative methods showed that the landforms conditioned the spatial pattern of soil attributes.
\end{abstract}

Key words: multivariate analysis, precision agriculture, hybrid mapping, soil-landscape relationships 


\section{INTRODUÇÃO}

As informações obtidas por meio do mapeamento da variabilidade dos atributos dos solos são utilizadas no planejamento das práticas de manejo para conservação do solo e da água (Mello et al., 2006; Silva et al., 2008; Sanchez et al., 2009; Vaezi et al., 2010), produção de culturas agrícolas (Sanchez et al., 2005; Siqueira et al., 2010b; Mann et al., 2010), estudos relacionados à gênese (Camargo et al., 2008a), otimização amostral (Montanari et al., 2005), emissão de $\mathrm{CO}_{2}$ (Panosso et al., 2009; La Scala Júnior et al., 2010; Brito et al., 2010; Mendonça et al., 2011; Merbold et al., 2011). Pode-se identificar, por meio deste mapeamento, áreas com maior homogeneidade dos atributos dos solos. Segundo Siqueira et al. (2010a), a identificação dessas regiões, chamadas áreas de manejo específico, garante a localização de limites mais precisos entre áreas distintas permitindo, assim, que técnicas agronômicas possam ser transferidas com facilidade e economia, para ambientes semelhantes.

Segundo Legros (2006), cinco procedimentos podem ser utilizados na identificação e mapeamento de solos e seus atributos: (a) mapeamento pela similaridade de diferentes pedons (mapeamento por malha de observações); (b) mapeamento livre ou categórico, no qual são utilizados conceitos de relação solo-paisagem para definir os locais de amostras; (c) mapeamento utilizando-se análise geoestatística; (d) mapeamento através da lógica fuzzy (e) classificação pela capacidade de uso da terra.

Recentemente, alguns pesquisadores (Cunha et al., 2005; Campos et al., 2007; Camargo et al., 2008a; Souza et al., 2009) têm proposto novas abordagens para caracterizar os solos e seus atributos utilizando, simultaneamente, métodos determinísticos (similaridade entre pedons, modelos de paisagem) e classificação numérica (geoestatística e análise multivariada). A união desses métodos gera um novo tipo de mapeamento, denominado híbrido. Conforme McBratney et al. (2000) esse tipo de mapeamento melhora o conhecimento da distribuição dos solos e seus atributos ao longo da paisagem (variabilidade espacial) e da precisão e da qualidade da informação, visto que diminui a subjetividade apresentada nos métodos tradicionais.

O uso de ferramentas, como o modelo digital de elevação (MDE) (Smith et al., 2006; Chagas et al., 2010), geoestatística (Campos et al., 2009) e de análises multivariadas (Siqueira et al., 2010b; Martín et al., 2010), são cada vez mais comuns em ciências agrárias e auxilia na identificação dessas áreas. Os referidos métodos de análises promovem uma visão espacial e integrada de todos os atributos ambientais, que permita uma substituição da subjetividade dos métodos de mapeamentos convencionais para mapeamentos mais objetivos com métodos quantitativos. O MDE é uma ferramenta útil no mapeamento de solo e sua distribuição na relação paisagem vem-se destacando em pesquisas sobre mapeamento, tornando-o mais acurado não dispensando, no entanto, o apoio de campo (Sousa Júnior \& Demattê, 2008).

De forma geral, o desenvolvimento agrícola nos trópicos necessita da caracterização e mapeamento precisos dos solos, antes de gerar recomendações adequadas de manejo (Shepherd
$\&$ Walsh, 2007). No Brasil, os primeiros mapeamentos poucos detalhados dos solos criaram uma falsa idéia de uniformidade dos seus atributos (Buol, 1990). Na classe taxonômica dos Latossolos, considerados solos mais intemperizados, portanto mais estáveis e homogêneo, vários pesquisadores têm encontrado variabilidade dos atributos mineralógicos (Camargo et al., 2008b), morfológicos (Souza et al., 2006b), físicos e químicos (Souza et al., 2006b).

No mapeamento mais detalhado dos atributos dos solos, Camargo et al. (2010), encontraram relação dos atributos físicos com as formas do relevo. Barbieri et al. (2009) relatam sobre a relação entre curvaturas de relevo, mineralogia da argila e adsorção de fósforo em Argissolo. Sanchez et al. (2009) concluíram que a magnitude da variabilidade dos atributos dos solos é mais influenciada pela forma do relevo que pela erosão. Esses autores afirmam que os sistemas de preparo provocam redução da espessura dos horizontes A+E incorporando, assim, uma variabilidade diferenciada de tais atributos ao longo de uma vertente. Assim, o mapeamento híbrido pode ser uma das soluções para a caracterização detalhada dos atributos dos solos em estudos de causa e efeito para adoção de programas de agricultura de precisão e implementação de planos adequados de manejo.

Desta maneira, o objetivo do presente trabalho foi caracterizar a variabilidade espacial de atributos físicos e químicos de Latossolos e Argissolos utilizando-se classificação numérica e o modelo digital de elevação com a finalidade de identificar um padrão de variabilidade diferenciada, condicionada pelas formas do relevo.

\section{Material e MÉTOdos}

\section{Caracterização do meio físico}

O experimento foi realizado em duas áreas localizadas no município de Jaboticabal, na parte nordeste do estado de São Paulo. As coordenadas geográficas são $21^{\circ} 17^{\prime}$ de latitude sul e $48^{\circ} 10^{\prime}$ de longitude oeste (área de Latossolo) e $20^{\circ} 56^{\prime}$ de latitude sul e $48^{\circ} 56^{\prime}$ longitude oeste (área de Argissolo), com altitude média de $600 \mathrm{~m}$. O clima da região é classificado, segundo Koppen, do tipo (CWa), predominando as chuvas de verão, com inverno relativamente seco. A temperatura média do mês mais quente é superior a $22^{\circ} \mathrm{C}$ e a do mês mais frio, inferior a $18^{\circ} \mathrm{C}$, sendo a precipitação anual de $1.440 \mathrm{~mm}$. Os materiais de origem dos solos estão relacionados ao arenito do grupo Bauru, formação Adamantina.

\section{Descrições morfológicas e obtenção de amostras}

Foram abertas seis trincheiras, sendo três nas duas pedoformas, nas quais foram realizadas: descrição morfológica, coleta de amostras e identificação dos horizontes $\mathrm{A}_{1}, \mathrm{Bw}_{2}$ e Bt dos solos, conforme Santos et al. (2005); também foram classificados como Latossolo Vermelho distrófico típico e Argissolo Vermelho eutrófico abruptico, segundo critérios estabelecidos pelo SiBCS (EMBRAPA, 2006), denominados como Latossolo e Argissolo, respectivamente.

As regiões foram percorridas para identificação dos modelos da paisagem; em seguida, escolhidas duas áreas sob o cultivo 
de cana-de-açúcar com o mesmo histórico de manejo, caracterizadas por possuírem pedoformas distintas, segundo classificação de Troeh (1965), convexa para o Latossolo e linear para o Argissolo, informação enriquecida pelo Modelo de elevação digital, conforme (Figura 1).

$\mathrm{Na}$ área do Latossolo (158 ha) foram retiradas 53 amostras e na área do Argissolo (172 ha) 57 amostras, na profundidade de 0,0-0,5 m. Todos os pontos foram georreferenciados com auxílio de um Sistema de Posicionamento Global (GPS).

\section{Métodos de laboratório}

As amostras dos horizontes genéticos das trincheiras dos solos forma submetidas a ataque sulfúrico (EMBRAPA, 1997).

Os atributos físicos dos solos foram determinados pelo método da pipeta, utilizando-se solução de $\mathrm{NaOH} 0,1 \mathrm{~mol} \mathrm{~L}^{-1}$, como dispersante químico e agitação mecânica em aparato de baixa rotação, durante $16 \mathrm{~h}$, obedecendo à metodologia proposta por (EMBRAPA, 1997). Após secagem e pesagem a fração areia foi subdividida em cinco subfrações, ou seja: areia muito grossa (AMG), areia grossa (AG), areia média (AM), areia fina (AF) e areia muito fina (AMF), em peneiras de 2-1 $\mathrm{mm}$; 1-0,5 mm; 0,5-0,25 mm;0,25-0,10 mm e 0,10-0,05 mm, respectivamente. A fração argila foi separada por sedimentação, conforme a Lei de Stokes; enfim, a fração areia por tamisação e a fração silte determinada por diferença.

A determinação dos atributos químicos do solo: Cálcio, Magnésio e Alumínio foi realizada utilizando-se o método de extração com $\mathrm{KCl} 1 \mathrm{~mol} \mathrm{~L}^{-1}$, titulado com EDTA 0,01 mol L-1 enquanto os teores de Fósforo e Potássio foram extraídos com $\mathrm{H}_{2} \mathrm{SO}_{4} 0,0125 \mathrm{~mol} \mathrm{~L}^{-1}+\mathrm{HCl} 0,05 \mathrm{~mol} \mathrm{~L}^{-1}$, embora o potássio tenha sido determinado por fotometria de chama e o fósforo por espectrometria de absorção atômica, conforme (Malavolta et al., 1997) e o $\mathrm{H}+\mathrm{Al}$ foi determinado a partir do $\mathrm{pH}$ SMP, conforme (Raij et al., 1987). O pH foi determinado potenciometricamente utilizando-se a relação de 1:2,5 de solo em $\mathrm{CaCl}_{2}$ 0,01 $\mathrm{mol} \mathrm{L}^{-1}$ e em KCl $1 \mathrm{~mol} \mathrm{~L}^{-1}$, de conformidade com (Camargo et al., 1986). A soma de bases (SB) foi calculada pela soma de $\mathrm{Ca}^{2+}, \mathrm{Mg}^{2+}, \mathrm{K}^{+}$e a capacidade de troca catiônica total (T) foi obtida pela soma da $(\mathrm{SB})+\mathrm{H}^{+}$e $\mathrm{Al}^{3+}$, sendo que a percentagem de saturação por bases $(\mathrm{V} \%)$ foi calculada por $\mathrm{V} \%=100 \mathrm{x} .(\mathrm{SB}) / \mathrm{T}$.

\section{Geração do modelo digital de elevação (MDE)}

O MDE foi gerado a patir das coordenadas de X (Longitude), Y (Latitude) e Z (Altitude) dos pontos da malha já georreferenciados, a partir das quais foi utilizado o programa Surfer (Golden Software, 1999), já que tem, disponíveis, vários interpoladores, dentre eles: o inverso da distância, kriging, vizinho mais próximo, método de Shepard, mínima curvatura, função de base radial, triangulação com interpolação linear, sendo que o interpolador utilizado neste trabalho foi a krigagem ordinária.

O MED é uma representação digital da superfície que permite uma rápida e útil análise topográfica de uma área de interesse, tal como entender variáveis ligadas à altitude (vertentes, orientações, limites de bacias hidrográficas, dinâmica do fluxo

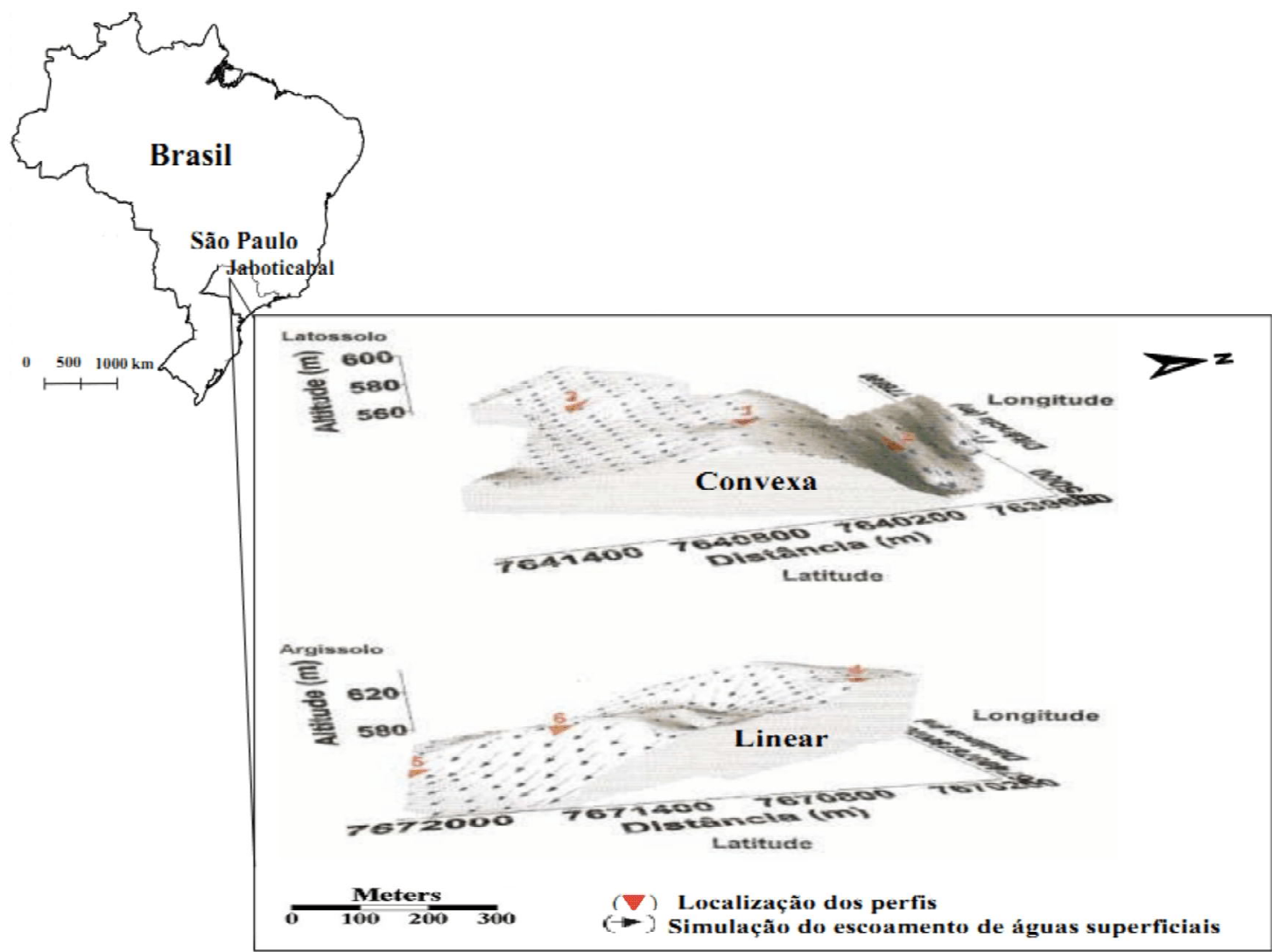

Figura 1. Área de localização e modelo digital de elevação (MDE), ilustrando as formas do relevo (Troeh, 1965), e localização dos perfis do solo e da simulação do fluxo de água das superfícies 
de água entre outros). Quando associado aos mapas de variabilidade, este modelo pode configurar-se como importante ferramenta para visualização e entendimento das relações de causa e consequência da distribuição espacial dos atributos dos solos (Hammer et al., 1995).

\section{Análise de agrupamento hierárquico (Cluster Analysis)}

Os atributos químicos e físicos dos solos foram submetidos à análise de agrupamento por método hierárquico, também conhecido como análise de "Cluster", segundo a metodologia proposta por Sneath \& Sokal (1973), com o objetivo de dividir os elementos da amostra em grupos, de forma que os elementos pertencentes a um mesmo grupo sejam similares entre si com relação às variáveis consideradas e os elementos de grupos diferentes sejam heterogêneos em relação a essas mesmas características (Webster \& Oliver, 1990). Esta técnica se inicia com a padronização dos dados para que cada variável tenha seus escores normalizados para o padrão $Z(\mu=0 ; s=1)$, através da expressão:

$$
Y_{i j}=\frac{X_{i j}-\overline{X_{i}}}{S_{i}}
$$

em que:

$$
\begin{aligned}
& X_{\mathrm{ij}} \text { - o valor da j-ésima observação da i-ésima variável } \\
& \bar{X}_{\mathrm{i}} \text { - a média da variável } \mathrm{X}_{\mathrm{ij}} \\
& \mathrm{S}_{\mathrm{i}} \text { - desvio padrão da variável } \mathrm{X}_{\mathrm{ij}} \\
& \mathrm{Y}_{\mathrm{ij}} \text { - a j-ésima observação da i-ésima variável padronizada }
\end{aligned}
$$

A etapa seguinte foi calcular as distâncias de cada objeto em relação a todos os outros objetos, calculando-se as distâncias euclidianas para obter coeficientes de dissimilaridade, ou seja, quanto menor forem os valores mais semelhantes eles são, conforme (Hair et al., 2005). Obtiveramse as distâncias através da expressão:

$$
\mathrm{d}_{\mathrm{ik}}=\frac{1}{\sqrt{\mathrm{P}}}\left[\frac{\mathrm{P}}{\sum_{\mathrm{j}=1}\left(\mathrm{X}_{\mathrm{ij}}-\mathrm{X}_{\mathrm{kj}}\right)^{1 / 2}}\right]
$$

em que:

$$
\begin{aligned}
& \mathrm{d}_{\mathrm{ik}} \text { - distância euclidiana média entre o grupo i e k } \\
& \mathrm{P} \text { - número de variáveis } \\
& \mathrm{X}_{\mathrm{ij}} \text { - valor da variável j no ponto i do grid } \\
& \mathrm{X}_{\mathrm{kj}} \text { - valor da variável j no ponto k do grid }
\end{aligned}
$$

A estratégia de agrupamento utilizada para os atributos físicos e químicos foi o algoritmo de Average Linkage (UPGMA Unweighted Pair Group Method with Arithmetic Averages) em que o número de grupos formados pode ser definido de diversas formas, como: a assistida ou "a sentimento" (Johnson \& Wichern, 1992; Mardia et al., 1997) cujo pesquisador pode especificar o nível de agrupamento de acordo com um critério de fácil interpretação. Assim foi escolhida, no presente estudo, uma distância euclidiana de 6,0 para atributos físicos e 6,75 para atributos químicos, em ambas as áreas. Os cálculos foram processados no software Statistica versão 5.5 (Statsoft, 1999). Foram confeccionados, a partir dos grupos definidos, os mapas de distribuição espacial por meio do programa Surfer (Golden Software, 1999), em que cada ponto pertencente a um mesmo grupo foi colocado na mesma classe.

\section{RESULTADOS E DISCUSSÃO}

\section{Morfologia, dados granulométricos e químicos dos perfis dos solos}

A descrição dos perfis relevou pequena variação na espessura dos horizontes $A_{1}$ e $B_{w 2}$ e $A_{1}$ e $B_{t 2}$ do Latossolo e Argissolo, respectivamente (Tabela 1), porém a espessura do horizonte A dos Argissolos é relativamente menor podendo ser atribuída à posição desses solos na paisagem, em declive mais acentuado.

O matiz de todos os horizontes, exceto do horizonte $A_{1}$ do Perfil 1 permaneceu em 2,5YR para os Latossolos e de 2,5 YR a 7,5 YR nos Argissolos, em virtude dos maiores teores de $\mathrm{F}_{2} \mathrm{O}_{3}$ desses Latossolos estarem em ambiente com melhor drenagem como consequiência do grande poder pigmentante da hematita, apresentando coloração avermelhada em detrimento da formação da goethita (Barbosa et al., 2009).

Os valores de Ki na (Tabela, 1) indicam que nos perfis localizados em áreas com melhor aeração há evolução direcionada à formação de minerais 1:1, ocasionada por perdas de Si (dessilicatização) pelo intemperismo dos minerais; os valores de Ki diminuem (Santos et al., 2010).

Como os solos apresentaram valores de Ki próximos possuem graus de intemperismo semelhantes, sendo assim, a variabilidade dos atributos químicos e físicos pode, então, ser melhor explicada pelo fluxo de água é condicionado pelo relevo (Coringa \& Weber, 2008).

Ao longo dos perfis dos solos, os valores de $\mathrm{pH}$ em $\mathrm{CaCl}_{2}$ variam de 3,8 a 5,1, sendo considerados, portanto, com acidez muito alta a alta (EMBRAPA, 2006). Isto, segundo (Rolim Neto et al., 2009), pode ser explicado pelo intenso uso agrícola dos solos e por sua posição de topo na paisagem e, ainda, em virtude desta condição topográfica de topo plano favorecer maior lixiviação de seus cátions trocáveis, resultando no acúmulo de $\mathrm{H}^{+}$e $\mathrm{Al}^{3+}$ no complexo de troca. Tal afirmação é corroborada pelo baixo valor $\mathrm{V} \%$ e alto valor $\mathrm{m} \%$ desses solos.

A soma de bases verificada nas camadas superficial e subsuperficial de cada um dos solos estudados, variou de 8,5 a 25,5 $\mathrm{mmol}_{\mathrm{c}} \mathrm{kg}^{-1}(\mathrm{~A} 1)$ e 2,5 a $13,5 \mathrm{mmol}_{\mathrm{c}} \mathrm{kg}^{-1}\left(\mathrm{~B}_{\mathrm{w} 2}\right)$ para o Latossolo e 6,3 a 15,6 mmol $_{\mathrm{c}} \mathrm{kg}^{-1}\left(\mathrm{~A}_{1}\right)$ e 19,5 mmol $_{\mathrm{c}} \mathrm{kg}^{-1}$ a 27,5 $\mathrm{mmol}_{\mathrm{c}} \mathrm{kg}^{-1}\left(\mathrm{~B}_{\mathrm{t} 2}\right)$ para o Argissolo. Referidos valores são concordantes com a saturação por bases dos horizontes $\mathrm{A}_{1} \mathrm{e}$ $\mathrm{B}_{\mathrm{w} 2}$ dos Latossolos variando de 17 a $45 \%$ e 8 a $51 \%$, respectivamente, sendo considerados de muito baixa a baixa (EMBRAPA, 2006). Para o Argissolo a saturação por bases no horizonte $\mathrm{A}_{1}$ variou de 12 a $25 \%$ e o $\mathrm{B}_{\mathrm{t} 2}$ de 38 a $57 \%$. Apesar dos solos apresentarem os coeficientes de Ki próximos, o Latossolo apresentou maiores teores de $\mathrm{F}_{2} \mathrm{O}_{3}$, porque há uma variação 
Tabela 1. Características morfológicas, granulométricas e químicas dos perfis do Latossolo e A rgissolo

\begin{tabular}{|c|c|c|c|c|c|c|c|c|c|c|c|c|}
\hline Horizonte & $\begin{array}{l}\text { Profundidade } \\
\qquad(\mathrm{cm})\end{array}$ & Cor úmida & Areia & $\begin{array}{l}\text { Silte } \\
\mathrm{g} \mathrm{kg}^{-1}\end{array}$ & Argila & $\begin{array}{c}\mathrm{pH} \\
\mathrm{CaCl}_{2}\end{array}$ & $\begin{array}{l}\mathrm{pH} \\
\mathrm{H}_{2} \mathrm{O}\end{array}$ & $\begin{array}{l}\mathrm{pH} \\
\mathrm{KCl}\end{array}$ & $\mathrm{PCZ}^{(1)}$ & V\%(2) & $\% \mathrm{Fe}_{2} \mathrm{O}_{3}$ & $\mathbf{K i}^{(3)}$ \\
\hline \multicolumn{13}{|c|}{ Perfil 1 (Latossolo Vermelho Distrófico típico, textura argilosa, A proeminente) } \\
\hline$A_{1}$ & $0-27$ & $5 Y R 4 / 4$ & 680 & 30 & 290 & 3,9 & 5,10 & 4,09 & $-0,92$ & 17 & 5,3 & 1,4 \\
\hline $\mathrm{B}_{\mathrm{W} 2}$ & $126-162+$ & $2,5 Y R 4 / 4$ & 615 & 45 & 340 & 3,8 & 4,68 & 4,34 & $-0,34$ & 8,0 & 5,7 & 1,5 \\
\hline \multicolumn{13}{|c|}{ Perfil 2 (Latossolo Vermelho Distrófico câmbico, textura argilosa, A proeminente) } \\
\hline$A_{1}$ & $0-28$ & $2,5 Y R 3 / 4$ & 665 & 65 & 270 & 3,9 & 5,06 & 4,25 & $-0,81$ & 19 & 6,7 & 1,1 \\
\hline $\mathrm{B}_{\mathrm{W} 2}$ & $133+$ & $2,5 Y R 4 / 4$ & 573 & 57 & 370 & 5,1 & 6,12 & 6,12 & 0,00 & 51 & 7,8 & 1,0 \\
\hline \multicolumn{13}{|c|}{ Perfil 3 (Latossolo Vermelho Distrófico típico, textura argilosa, A proeminente) } \\
\hline$A_{1}$ & $0-16$ & $2,5 Y R 3 / 4$ & 638 & 62 & 300 & 4,6 & 5,88 & 4,61 & $-1,27$ & 45 & 5,1 & 1,3 \\
\hline $\mathrm{B}_{\mathrm{W} 2}$ & $97-151$ & $2,5 Y R \quad 4 / 4$ & 480 & 90 & 430 & 3,8 & 4,63 & 4,18 & $-1,15$ & 16 & 7,9 & 1,6 \\
\hline \multicolumn{13}{|c|}{ Perfil 4 (Argissolo Vermelho-Amarelo Eutrófico abrúptico, textura argilosa, A moderado) } \\
\hline$A_{1}$ & $0-26$ & 5YR $3 / 2$ & 803 & 67 & 130 & 4,0 & 5,34 & 4,06 & $-1,28$ & 25 & 2,9 & 0,9 \\
\hline $\mathrm{B}_{\mathrm{t} 2}$ & $92-147$ & $5 Y R \quad 4 / 6$ & 571 & 79 & 350 & 5,0 & 5,98 & 5,01 & $-0,97$ & 56 & 4,6 & 2,9 \\
\hline \multicolumn{13}{|c|}{ Perfil 5 (Argissolo Vermelho-Amarelo Distrófico abrúptico, textura média, A proeminente) } \\
\hline$A_{1}$ & $0-28$ & $7,5 Y R 3 / 2$ & 810 & 40 & 150 & 3,9 & 3,9 & 3,9 & $-1,02$ & 17 & 2,5 & 1,4 \\
\hline $\mathrm{B}_{\mathrm{t} 2}$ & $105-153$ & 5YR 4/6 & 673 & 67 & 260 & 5,1 & 5,1 & 5,1 & $-0,81$ & 57 & 4,0 & 1,5 \\
\hline \multicolumn{13}{|c|}{ Perfil 6 (Argissolo Vermelho Distrófico típico, textura argilosa, A proeminente) } \\
\hline$A_{1}$ & $0-36$ & $7,5 Y R 3 / 4$ & 813 & 27 & 160 & 3,8 & 4,06 & 3,9 & $-0,70$ & 12 & 2,7 & 1,7 \\
\hline $\mathrm{B}_{\mathrm{t} 2}$ & $65-110$ & $2,5 Y R 4 / 6$ & 619 & 61 & 320 & 4,5 & 5,21 & 4,59 & $-0,62$ & 38 & 4,3 & 1,1 \\
\hline
\end{tabular}

(1) $\mathrm{PCZ}\left(\right.$ ponto de carga zero) $\left.=2 \mathrm{pHKCL}-\mathrm{pHH}_{2} \mathrm{O}\right) ;{ }^{(2)} \mathrm{V} \%$ - saturação por bases; ${ }^{(3)} \mathrm{Ki}$ - índice de intemperismo do solo

consistente nos valores dos atributos cristalográficos da hematita e goethita, de acordo com a profundidade do solo e a posição do perfil na topossequência (Ghidin et al., 2006).

Ao conhececimento dos valores de PCZ é fundamental, visto que este pode inferir certas propriedades relacionadas à pedogênese, permitindo diferenciar os solos. Nota-se que o PCZ apresentou valores negativos e bastante próximos em cada perfil do solo, exceto no horizonte $\mathrm{B}_{\mathrm{w} 2}$ do Latossolo, que foi 0,0 .

Os resultados da análise de agrupamento são apresentados na Figura 2, em que nos dendogramas do Latossolo nas Figuras 2 (A e C) tanto para os atributos físicos quanto para os químicos, houve formação de um número maior de grupos em relação aos dendogramas do Argissolo Figuras 2 (B e D). Mesmo sendo a classe dos latossolos considerada a mais intemperizada e, portanto, a mais homogênea, apresentou maior variabilidade dos atributos do solo, indicando que a variabilidade está mais associada com a forma da paisagem do que com a classe taxonômica.

Desta forma, estudos de solo mais detalhados com utilização da análise de Cluster podem revelar informações pedológicas importantes que não são aparentemente observadas quando os pedons são classificados de maneira individualizada na paisagem, fugindo do conceito de solo como corpo natural (Young \& Hammer, 2000).

As diferenças da variabilidade espacial de atributos físicos e químicos nas diversas classes de solo estão associadas às formas do relevo, sendo este o principal responsável pelas maiores variabilidades na área convexa (Latossolo) em relação à área linear (Argissolo); resultados semelhantes também foram encontrados por (Souza et al., 2009; Souza et al., 2004; Montanari et al., 2005), estudando a influência da forma da paisagem sob cultivo de cana-de-açúcar.

Desta maneira, estudos sobre as relações da variabilidade dos solos e seus atributos com métodos geomorfológicos, como as formas do relevo a partir do modelo digital de elevação, podem ser utilizados como ferramentas de auxílio na avaliação pedológica, já que mencionados métodos não consideram os limites pré-estabelecidos pela taxonomia mas seguem os limites dos solos como corpos naturais.

Assim, a partir das áreas mapeadas do Latossolo e do Argissolo, ilustradas nas Figuras 3 (A e B), percebe-se a grande influência da paisagem, por consequência do fluxo de água, no ambiente de formação de cada classe de solo, sendo a maioria da homogeneidade granulométrica do solo de compartimentos mais elevados (chapadas), com perfis invariantemente muitos argilosos, indicando independência do substrato rochoso com a distribuição condicionada à variação do regime hídrico ao longo da topossequencia (Motta et al., 2002).

Esta dinâmica condiciona um ambiente de alta instabilidade, o que pode ser confirmado pela maior formação de grupos, tanto para atributos físicos quanto para químicos do solo.

Observa-se, na pedoforma linear (Argissolo) tendência à estabilização, o que é caracterizado pelo menor número de grupos formados. Por este motivo, os aspectos topográficos do terreno podem ser bons indicadores da variação dos atributos do solo haja vista que esta variabilidade é causada por pequenas alterações do declive que influenciam o transporte e o armazenamento de água dentro do perfil do solo (Sanchez et al., 2009; Campos et al., 2010), as quais podem ser bem observadas pelo MDE, na Figura 1.

Nota-se então, associando o padrão dos grupos formados com base na análise de agrupamento com o MDE, que o Latossolo em pedoforma convexa apresentou maior variabilidade espacial dos atributos do solo que o Argissolo em pedoforma linear. Esses resultados corroboram com os de Montanari et al. (2008), que também encontraram maior variação dos atributos do solo na forma convexa em latossolo em relação à forma línea, em Argissolo.

Montanari et al. (2005) constataram, estudando a variabilidade espacial de duas formas do relevo que, apesar de 
A.

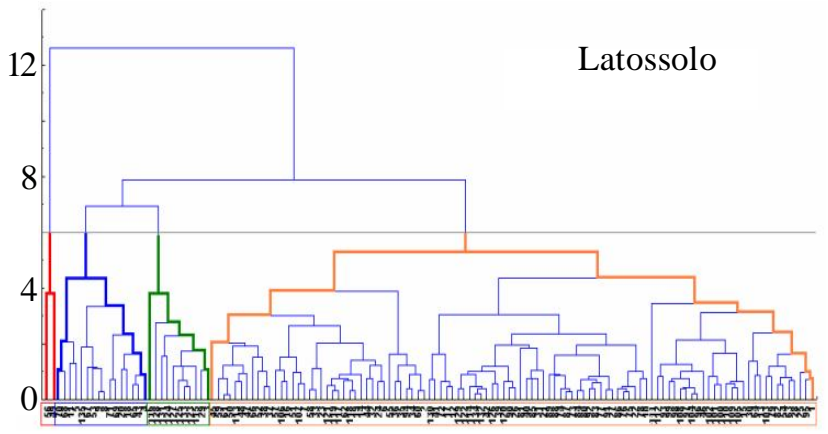

B.

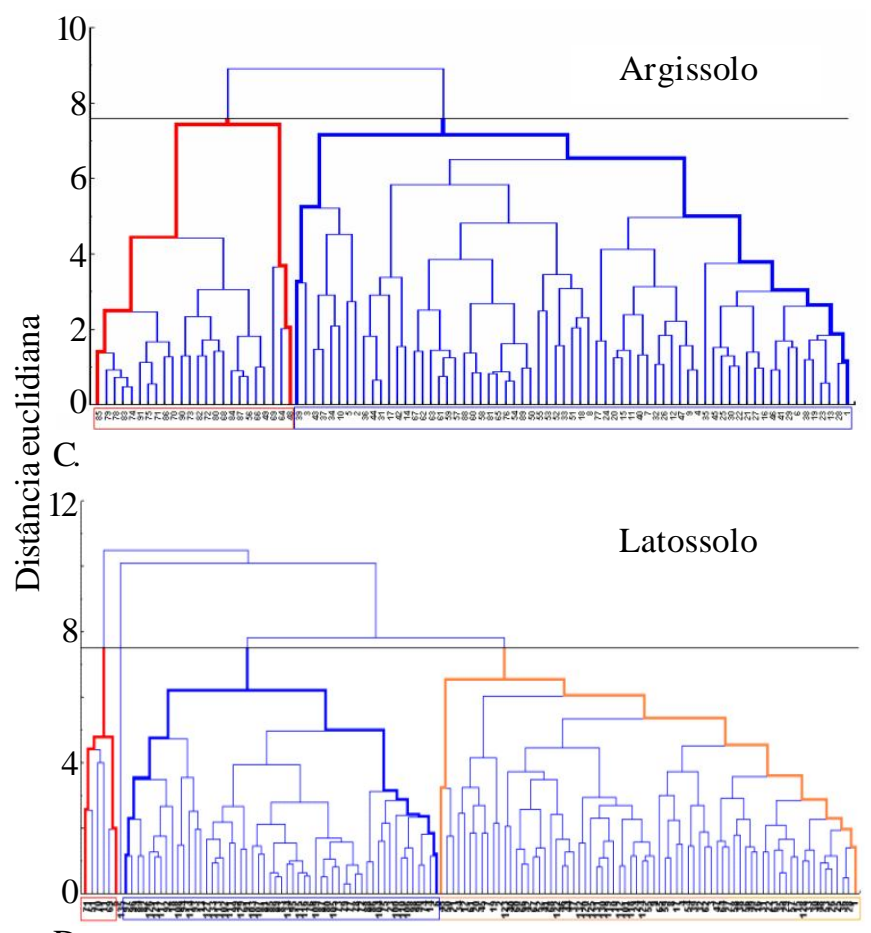

D.

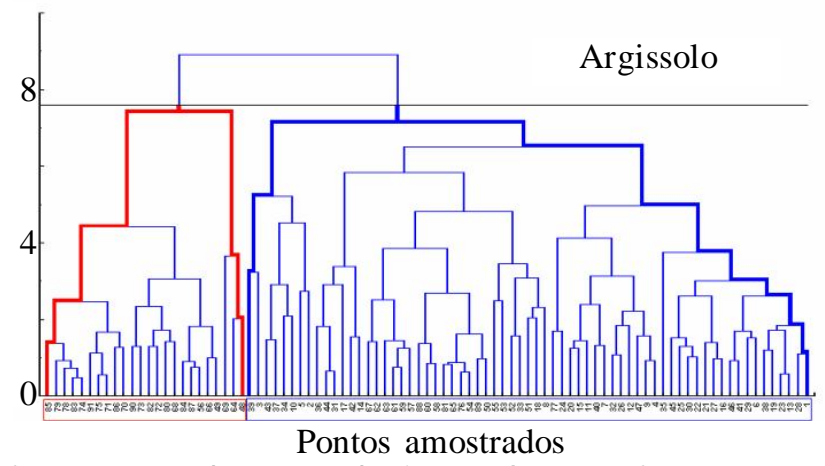

Figura 2. D endogramas das inter-relações referentes aos atributos físicos (A) e (B) e aos químicos (C) e (D) pela distância euclidiana média

os latossolos serem considerados solos antigos, altamente intemperizados, dependendo na posição da paisagem, podem apresentar características não próprias desta classe. Esses resultados se devem à variabilidade espacial dos atributos do solo relacionados com as variações do relevo, corroborando com os de Siqueira et al. (2010b) que, estudando as formas de relevo para predizer a variabilidade de atributos do solo e plantas com a geoestatística e análise multivariada de correlação canônica, observaram que é o relevo que condiciona o comportamento espacial dos atributos físicos e químicos do solo e esses, por sua vez, interferem na produção e na qualidade das plantas de laranja.

Visto que os dendogramas dos atributos físicos e químicos concordam com as relações de Jenny (1941) de que as características do solo são formadas com o tipo de relevo, em que solos planos apresentam maior umidade em relação a terras altas e solos úmidos e mais profundos, tenderão ser mais estáveis (homogêneos) em relação a terras altas devido à menor infiltração nesses solos. Deste modo, a forma do relevo pode auxiliar não apenas na definição de esquemas amostrais mas também no mapeamento de zonas de manejo físico e químico do solo (Montanari et al., 2005; Souza et al., 2006a; Leão et al., 2011).

De acordo com Montanari et al. (2005), as pedoformas em áreas de mesma classe de solo e mesmo histórico de manejo de cana-de-açúcar durante vários anos, influenciam na variabilidade dos atributos químicos dos solos de maneira sistemática, corroborando com Souza et al. (2004; 2006b), que mostram haver maior variabilidade de atributos químicos e físicos em áreas de formas côncavas e convexas, em relação às formas lineares, independentemente do histórico de manejo dessas áreas. O conhecimento desses atributos topográficos derivados de MDE, resulta em unidades preliminares de mapeamento do solo, seguindo a recomendação de Moore et al. (1993) que indicam a utilização dessa representação da superfície por meio dos referidos modelos, tanto para amostragem quanto para definição de pedoformas.

Irvin et al. (1997) também demonstraram a utilização de atributos derivados de MDE e sua classificação para a delimitação automática de geoformas e confirmaram que o uso de métodos de classificação numérica para sua identificação provou ser uma técnica promissora, tanto que Ippoliti et al. (2005) deduziram que o principal mérito do método descrito na análise digital do terreno é a maior eficiência obtida no trabalho de campo, após a realização de uma classificação digital preliminar, em virtude de um modelo de ocorrência de solos previamente conhecido; mas se deve, ainda, promover ajustes, sendo necessário considerar outros componentes da paisagem ou variáveis ambientais recomendando a integração das variáveis ambientais na predição das pedoformas, por meio de métodos numéricos que possuam a característica de integração de dados.

Neste paradigma se deve traçar um panorama geral sobre os métodos de mapeamento de solos e/ou de suas propriedades tal como sobre as principais técnicas quantitativas usadas levando a concluir, pelo exposto, quanto à necessidade de se adotar um modelo híbrido (McBratney et al., 2000), combinando os dois métodos através da relação entre os fatores ambientais topográficos do MDE que influenciam os processos multivariados de formação do solo, descritos por Jenny (1941), com métodos geoestatísticos, e também multivariados (análise de Cluster), usados para a interpolação espacial dos valores preditos ou de seus resíduos (Odeh et al., 1995).

Neste sentido, a aplicação conjunta da análise de agrupamento com os atributos topográficos a partir de MDE 
Latossolo - Parâmetros físicos

A.

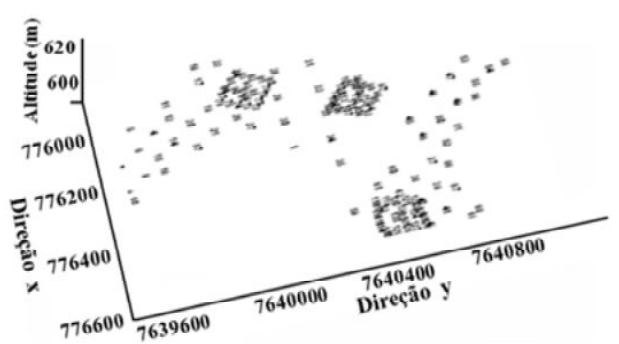

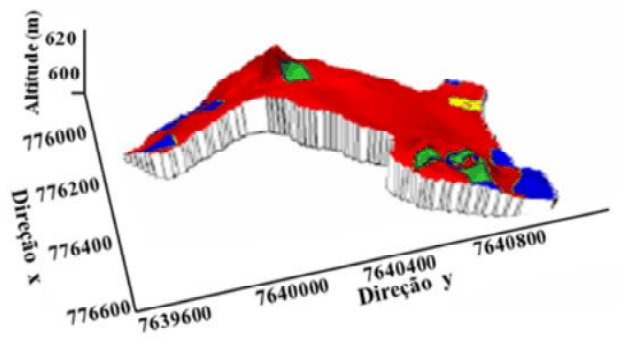

Argissolo - Parâmetros físicos
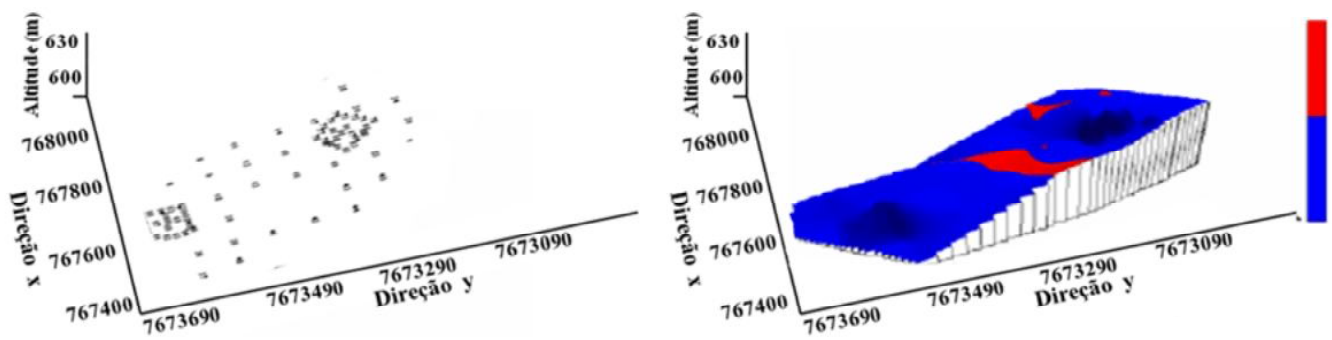

Latossolo - Parâmetros químicos

B.
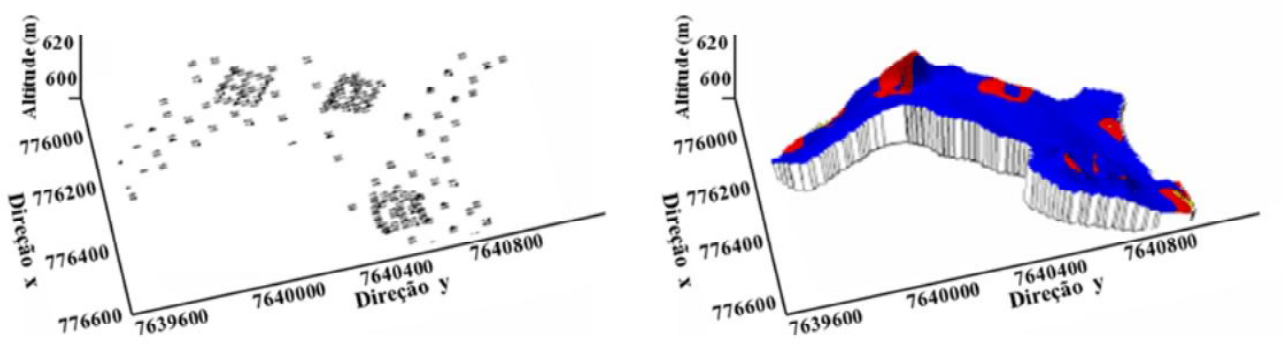

Argissolo - Parâmetros químicos
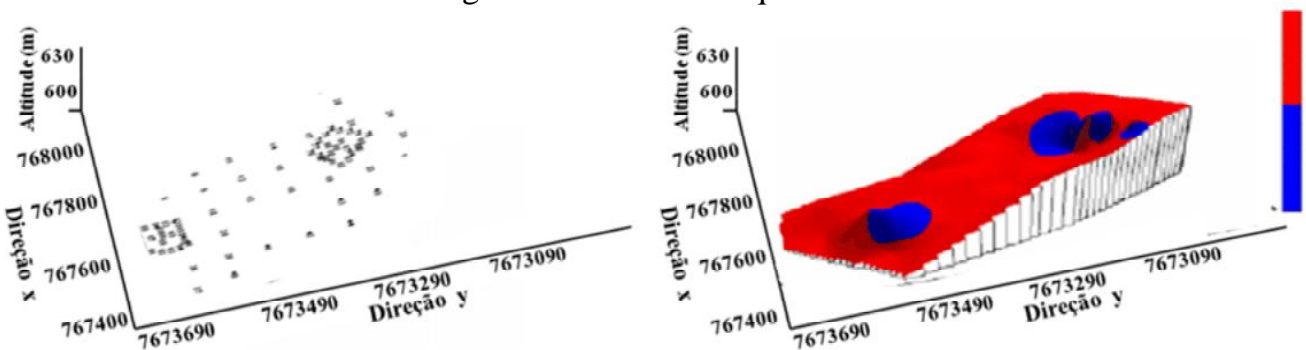

Figura 3. M al has amostrais e áreas mapeadas com a distribuição espacial dos atributos físicos e químicos correspondentes aos dendogramas das diferentes classes de solos (Latossolo e Argissolo)

indica a viabilidade da utilização das formas do relevo como critério discriminante dos padrões de variabilidade espacial dos atributos químicos e físicos dos solos diferenciados, tornando-se evidente que Latossolos em pedoforma convexa são mais heterogêneos espacialmente em comparação com o Argissolo em pedoforma linear, o que concorda com Camargo et al. (2008a) e Montanari et al. (2008). O conhecimento desses padrões espaciais e da variância dos atributos é fundamental para definir uma estratégia de amostragem espacial, que é o fator que mais influencia a eficiência e o custo da produção agrícola (Groenigen et al., 1999).

Verificou-se, portanto, um padrão de variabilidade espacial diferenciada nas partes do terreno dos dois solos, de tal forma que é imprescindível estabelecer um plano de amostragem com intensidade diferenciada, tanto que Vasat et al. (2010) comprovaram que a amostragem do solo é um componente bastante significativo nos levantamentos de solos e, portanto, requer uma análise criteriosa ao incorporá-la, ao planejamento dos levantamento pedológicos.

Desta forma, Montanari et al. (2008) recomendam um número maior de amostras para o Latossolo inserido em pedoforma convexa, por apresentar maior variabilidade espacial dos atributos nessas áreas, sendo necessário a aplicação de técnicas de otimização amostral.

Portanto, esses resultados indicam que a análise de agrupamento e o MDE relacionados com as formas da paisagem, podem ser indicativo para o planejamento amostral, como discutido por Montanari et al. (2005), para o estudo de 
pedogênese (Camargo et al., 2008), constituindo-se de um poderoso instrumento de predição da variabilidade espacial quando associado à análise de agrupamento hierárquica.

\section{Conclusões}

1. A classificação numérica através da análise de agrupamento auxiliou na identificação da similaridade dos atributos físicos e químicos dos solos de diferentes locais entre diferentes classes taxonômicas e formas do relevo.

2. O conceito relação solo-paisagem e o modelo digital de elevação são ferramentas eficientes no mapeamento de áreas homogêneas podendo ser recomendadas para auxiliar na tomada decisões mais precisas no manejo diferenciado em relação à variabilidade das classes dos solos.

3. Os mapas da distribuição espacial mostraram que a classificação numérica e o modelo digital de elevação foram eficientes na identificação dos diferentes padrões de variabilidade espacial nas pequenas variações do relevo das classes de solo podendo transferir essas informações para áreas semelhantes ou reduzir os erros de previsão taxonômicos.

\section{LITERATURA CITADA}

Barbieri, D. M.; Marques Jr., J; Alleoni, L. R. F.; Garbuio, F. J.; Camargo, L. A. Hillslope curvature, clay mineralogy, and phosphorus adsorption in an Alfisol cultivated with sugarcane. Scientia Agrícola, v.66, p.819-826, 2009.

Barbosa, I. O.; Lacerda, M. P. C.; Bilich, M. R. Relações pedomorfogeológicas nas chapadas elevadas do distrito federal. Revista Brasileira de Ciência do Solo, v.33, p.13731383, 2009.

Brito, L. de F.; Marques Júnior, J.; Pereira, G. T.; La Scala, Jr. spatial variability of soil $\mathrm{CO}_{2}$ emission in different topography positions. Bragantia, v.69, p.19-27, 2010.

Buol, S. W. Suelos tropicales: classificación y características. In: Salinas, J. G.; Gouley, L. M. Sorgo para suelos ácidos. Cali: CIAT, 1990. p.49-62.

Camargo, L. A.; Marques Júnior, J.; Pereira, G. T. Spatial variability of physical attributes of an alfisol under different hillslope curvatures. Revista Brasileira de Ciência do Solo, v.34, p.617-630, 2010.

Camargo, L. A.; Marques Júnior, J.; Pereira, G. T.; Horvat, R. A. Variabilidade espacial de atributos mineralógicos de um latossolo sob diferentes formas de relevo. I - Mineralogia da fração argila. Revista Brasileira de Ciência do Solo, v.32, p.2269-2277, 2008a.

Camargo, L. A.; Marques Júnior, J.; Pereira, G. T.; Horvat, R. A. Variabilidade espacial de atributos mineralógicos de um latossolo sob diferentes formas do relevo. II - Correlação espacial entre mineralogia e agregados. Revista Brasileira de Ciência do Solo, v. 32, p.2279-2288, 2008b.

Camargo, O. A.; Moniz, A. C.; Jorge, J. A.; Valadares, J. M. A. S. Métodos de análise química, mineralógica e física de solos do Instituto Agronômico de Campinas. Campinas: Instituto Agronômico, 1986. 94p. Boletim Técnico, 106
Campos, M. C. C.; Marques Júnior, J.; Pereira, G. T.; Montanari, R.; Camargo, L. A. Relações solo-paisagem em uma litosseqüência arenito-basalto na região de Pereira Barreto, SP. Revista Brasileira de Ciência do Solo, v.31, p.519-539, 2007.

Campos, M. C. C; Marques Júnior, J.; Pereira, G. T.; Souza, Z. M.; Montanari, R. Planejamento agrícola e implantação de sistema de cultivo de cana-de-açúcar com auxílio de técnicas geoestatísticas. Revista Brasileira de Engenharia Agrícola e Ambiental, v.13, p.297-304, 2009.

Campos, M. C. C.; Ribeiro, M. R.; Souza Júnior, V. S.; Ribeiro Filho, M. R.; Costa, E. U. C. Segmentos de vertente e atributos do solo de uma toposseqüência na região de Manicoré, AM. Ciência Agronômica, v.41, p.501-510, 2010.

Chagas, C. S.; Fernandes Filho, E. I.; Rocha, M. F.; Carvalho Júnior, W.; Souza Neto, N. C. Avaliação de modelos digitais de elevação para aplicação em um mapeamento digital de solos. Revista Brasileira de Engenharia Agrícola e Ambiental, v.14, p.218-226, 2010.

Coringa, E. A. O.; Weber, O. L. S. Ponto de efeito salino nulo de Latossolos da microbacia Chico Nunes, Mato Grosso. Revista Brasileira de Ciência do Solo, v.32, p.441-448, 2008.

Cunha, P.; Marques Júnior, J.; Curi, N.; Pereira, G. T.; Lepsch, I. F. Superfícies geomórficas e atributos de latossolos em uma sequiência arenítico-basáltica da região de Jaboticabal (SP). Revista Brasileira de Ciência do Solo, v.29, p.81-90, 2005.

EMBRAPA - Empresa Brasileira de Pesquisa Agropecuária Centro Nacional de Pesquisa de Solos: Manual de métodos de análise de solo. 2.ed. Rio de Janeiro: Embrapa CNPS, 1997.212p.

EMBRAPA - Empresa Brasileira de Pesquisa Agropecuária. Centro Nacional de Pesquisa de Solos. Sistema Brasileiro de Classificação de Solos. Rio de Janeiro: Embrapa CNPS. 2006. 306p.

Ghidin, A. A.; Melo,V. F.; Lima,V. C.; Costa, J. M. J. L. Topossequiências de latossolos originados de rochas basálticas no paraná. II - Relação entre mineralogia da fração argila e propriedades físicas dos solos. Revista Brasileira de Ciência do Solo, v.30, p.307-319, 2006.

Golden Software Inc. (Golden, Estados Unidos). SURFER for Windows: realese 7.0: contouring and 3D surface mapping for scientist's engineers, user's guide. New York, 1999. 619p.

Groenigen, van J. W.; Siderius, W.; Stein, A. Constrained optimization of soil sampling for minimization of the kriging variance. Geoderma, v.87, p.239-59, 1999.

Hair, J. F.; Anderson, R. E.; Tatham, R. L.; Black, W. C. Análise multivariada de dados. 5.ed. Porto Alegre: Bookman, 2005. $595 \mathrm{p}$.

Hammer, R. D.; Young, N. C.; Wolenhaupt, T. L.; Barney T. L.; Haithcoate, T. W. Slope class maps form soil survey and digital elevation models. Soil Science Society American Journal, v.59, p.509-519, 1995.

Ippoliti, G. A.; Costa, L. M.; Schaefer, C. E. G. R.; Fernandes Filho, E. I.; Gaggero, M. R.; Souza, E. Análise digital do terreno: Ferramenta na identificação de pedoformas em microbacia na região de "Mar de Morros" (MG). Revista Brasileira de Ciência do Solo, v.29, p.269-276, 2005. 
Irvin, B. J.; Ventura, S. J.; Slater, B. K. Fuzzy and Isodata classification of landform elements from digital terrain data in Pleasant alley. Geoderma, v.7, p.137-154, 1997.

Jenny, H. Factors of soil formation: A system of quantitative pedology. McGraw-Hill: New York, 1941. 281p.

Johnson, R. A.; Wichern, D. W. Applied multivariate statistical analysis. 3.ed. New Jersey: Prantice Hall, 1992. 642p.

La Scala Jr., N; Mendonça, E. S.; Souza, J. V.; Panosso, A. R.; Simas, F. N. B.; Schaefer, C. E. R. G. Spatial and temporal variability in soil $\mathrm{CO}_{2}-\mathrm{C}$ emissions and relation to soil temperature at King George Island, Maritime Antarctica, Maritime Antarctica. Polar Science, v.4, p.479-487, 2010.

Leão, M. G. A.; Marques Júnior, J.; Souza, Z. M.; Siqueira, D. S.; Pereira, G. T. Terrain forms and spatial variability of soil properties in an area cultivated with citrus. Revista Brasileira de Engenharia Agrícola, v.31, p.643-651, 2011.

Legros, J. P. Mapping of the soil. Translated from french by Sarma, V. A. K. Enfield: New Hampshire. Science Publishers, 2006. 411p.

Mann, K. K.; Schumann, A. W.; Obreza, T. A.; Harris, W. G.; Shukla, S. Spatial variability of soil physical properties affecting Florida citrus production. Soil Science v.175, p.487499, 2010.

Mardia, A. K. V.; Kent. J. T.; Bibby, J. M. Multivariate analysis. London: Academic Press, 1997. 518p.

Malavolta, E.; Vitti, G. C.; Oliveira, S. A. Avaliação do estado nutricional das plantas: princípios e aplicações. 2.ed. Piracicaba: Associação Brasileira para Pesquisa da Potassa e do Fosfato, 1997. 319p.

Martin, M. Z.; Labbé, N.; André, N.; Wullschleger, S. D.; Harris, R. H.; Ebinger, M. H. Novel multivariate analysis for soil carbon measurement using Laser-Induced breakdown Spectroscopy. Soil Science Society of America Journal, v.74, p.87-93, 2010

McBratney, A. B.; Odeh. I. O. A.; Bishop, T. F. A.; Dunbar, M. S.; Shatar, T. M. An overview of pedometric techniques for use in soil survey. Geoderma, v.97, p.293-327, 2000.

Mello, G.; Bueno, C. R. P.; Pereira, G. T. Variabilidade espacial de perdas de solo, do potencial natural e risco de erosão em áreas intensamente cultivadas. Revista Brasileira de Engenharia Agrícola e Ambiental, v.10, p.315-322, 2006.

Mendonça, E. S.; La Scala, N; Panosso, A. R.; Simas, F.N.B.; Schaefer, C. E. R. G Spatial variability models of CO2 emissions from soils colonized with grass (Deschampsia antarctica) and moss (Sanionia uncinata) in Admiralty Bay, Maritime Antarctica. Antarctic Science (Print), v.23, p.27-33, 2011.

Merbold, L.; Ziegler, W.; Mukelabai, M. M.; Kutsch, W. L. Spatial and temporal variation of $\mathrm{CO} 2$ efflux along a disturbance gradient in a miombo woodland in Western Zambia. Biogeociences, v.8, p.147-164, 2011.

Montanari, R.; Marques Júnior, J.; Pereira, G. T.; Souza, Z. M. Forma da paisagem como critério para otimização amostral de latossolos sob cultivo de cana-de-açúcar. Pesquisa Agropecuária Brasileira, v.40, p.69-77, 2005.

Montanari, R.; Pereira, G. T.; Marques Júnior, J.; Souza, Z. M.; Pazeto, R. J.; Camargo, L. A. Variabilidade espacial de atributos químicos em Latossolo e Argissolo. Ciência Rural, v.38, p.1266-1272, 2008.
Moore, I. D.; Gessler, P. E.; Nielsen, G. A.; Peterson, G. A. Soil attribute prediction using terrain analysis. Soil Science Society American Journal, v.57, p.443-452, 1993.

Motta, P. E. F.; Carvalho Filho, A.; Ker, J. C. Relações solosuperfície geomórfica e evolução da paisagem em uma área do Planalto Central Brasileiro. Pesquisa Agropecuária Brasileira, v.37, p.869-878, 2002.

Odeh, I. O. A.; McBratney, A. B.; Chittleborough, D. J. Further results on prediction of soil properties from terrain attributes: Heterotopic cokriging and regression kriging. Geoderma, v.67, p.215-225, 1995.

Panosso, A. R.; Marques Júnior, J.; Pereira, G. T.; La Scala Jr, N. Spatial and temporal variability of soil $\mathrm{CO} 2$ emission in a sugarcane area under green and slash-and-burn managements. Soil \& Tillage Research, v.105, p.275-282, 2009.

Raij, B. van; Quaggio, J. A.; Cantarella, H.; Ferreira, M. E.; Lopes, A. S.; Bataglia, O. C. Análise química do solo para fins de fertilidade. Campinas: Fundação Cargill, 1987. 170p.

Rolim Neto, F. R. C.; Schaefer, C. E. G. R.; Fernandes Filho, E. I.; Corrêa, M. M.; Costa, L. M.; Parahyba, R. B. V.; Guerra, S. M. S.; Heck, R. Topolitossequências de solos do alto paranaíba: atributos físicos, químicos e mineralógicos. Revista Brasileira de Ciência do Solo, v.33, p.1795-1809, 2009.

Sanchez, R. B.; Marques Júnior, J.; Pereira, G. T.; Souza, Z. M. Variabilidade espacial de propriedades de Latossolo e da produção de café em diferentes superfícies geomórficas. Revista Brasileira de Engenharia Agrícola e Ambiental, v.9, p.489-495, 2005.

Sanchez, R. B.; Marques Júnior, J.; Souza, Z. M.; Pereira, G. T.; Martins Filho, M. V. Variabilidade espacial de atributos do solo e de fatores de erosão em diferentes pedoformas. Bragantia, v.68, p.1095-1103, 2009.

Santos, A. do C.; Pereira, M. G.; Anjos, L. H. C.; Bernini, T. de A.; Cooper, M.; Nummer, A. R.; Francelino, M. R. Gênese e classificação de solos numa topossequência no ambiente de mar de morros do médio Vale do Paraíba do Sul, RJ. Revista Brasileira de Ciência do Solo, v.34, p.1297-1314, 2010.

Santos, R. D.; Lemos R. C.; Santos, H. G.; Ker, J. C.; Anjos, L. H. C. Manual de descrição e coleta de solo no campo. 5.ed. Viçosa, Sociedade Brasileira de Ciência do Solo, 2005. 100p.

Shepherd, K. D.; Walsh, M. G. Infrared spectroscopy - Enabling an evidence based diagnostic survellance approach to agricultural and environmental management in developing countries: Journal of Near Infrared Spectroscopy, v.15, p.119, 2007.

Siqueira, D. S.; Marques Júnior, J.; Matias, S. S. R.; Barrón, V.; Torrent, J.; Baffa, O.; Oliveira, L. C. de. Predicting the properties of Brazilian Haplustalfs from magnetic susceptibility measurements. Soil Use and Management, v.26, p.425-431, 2010a.

Siqueira, D. S.; Marques Júnior, J.; Pereira, G. T. The use of landforms to predict the variability of soil and orange attributes. Geoderma, v.155, p.55-66, 2010b.

Silva, A. M.; Mello, C. R.; Curi, N. I.; Oliveira, P. M. Simulação da variabilidade espacial da erosão hídrica em uma subbacia hidrográfica de latossolos no sul de minas gerais. Revista Brasileira de Ciência do Solo, v.32, p.2125-2134, 2008. 
Smith, M. P.; Zhu, A. X.; Burt, J. E.; Stiles, C. The effects of DEM resolution and neighborhood size on digital soil survey. Geoderma, v.155, p.55-66, 2006.

Sneath, P. H.; Sokal, R. R. Numerical taxonomy: The principles and practice of numerical classification. San Francisco: W.H. Freeman, 1973. 573p.

Sousa Júnior, J. G. A.; Demattê, J. A. M. Modelo digital de elevação na caracterização de solos desenvolvidos de basalto e material arenítico. Revista Brasileira de Ciência do Solo, v.32, p.449-456, 2008.

Souza, Z. M.; Marques Júnior, J.; Cooper. M.; Pereira, G. T. Micromorfologia do solo e sua relação com atributos físicos e hídricos. Pesquisa Agropecuária Brasileira, v.41, p.487492, 2006a.

Souza, Z. M.; Marques Júnior, J.; Pereira, G. T. Spatial variability of the physical and mineralogical properties of the soil from the areas with variation in landscape shapes. Brazilian Archives of Biology and Technology, v.52, p.305-316, 2009.

Souza, Z. M.; Marques Júnior, J.; Pereira, G. T.; Barbieri, D. M. Variabilidade espacial da textura de um Latossolo Vermelho eutroférrico sob cultivo de cana-de-açúcar. Engenharia Agrícola, v.24, p.309-319, 2004.
Souza, Z. M.; Marques Júnior, J.; Pereira, G.; Montanari, R.; Campos, M. C. C. Amostragem de atributos químicos e físicos em área com variação nas formas do relevo. Científica, v.34, p.249-256, 2006 b.

Statsoft, Inc. Statistica for Windows - computer program manual. Tulsa: StatSoft, 1999.

Troeh, F. R. Landform equations fitted to contour maps. American Journal of Science, v.263, p.616-627, 1965.

Vaezi, A. R.; Bahrami, H. A.; Sadeghi, S. H. R.; Mahdian, M. H. Spatial variability of soil erodibility factor $(\mathrm{K})$ of the USLE in north west of iran. Journal of Agricultural Science and Technology, v.12, p.241-252, 2010.

Vašát, R.; Heuvelink, G. B. M.; Borùvka, L. Sampling design optimization for multivariate soil mapping. Geoderma, v.155, p.147-153, 2010.

Young, F. J.; Hammer, R. D. Defining geographic soil bodies by landscape position, soil taxonomy, and cluster analysis. Soil Science Society American Journal, v.64, p.989-998, 2000.

Webster, R.; Oliver, M. A. Statistical methods in soil and land resource survey. Oxford: Oxford University Press. 1990. $316 \mathrm{p}$. 